\title{
Los quirópteros de Chile: avances en el conocimiento, aportes para la conservación y proyecciones futuras
}

\section{Chilean bats: advances knowledge, contributions for conservation and future projections}

\author{
Cristina Sierra-Cisternas ${ }^{1,2} \&$ Enrique Rodríguez-Serrano2* \\ ${ }^{1}$ Programa de Magister en Ciencias, mención Zoología, Departamento de Zoología, Facultad de Ciencias Naturales y \\ Oceanográficas, Universidad de Concepción, Concepción, Chile. Apartado Postal Casilla 160-C, Concepción. \\ ${ }^{2}$ Laboratorio de Mastozoología, Departamento de Zoología, Facultad de Ciencias Naturales y Oceanográficas, Universidad de \\ Concepción, Concepción, Chile. Apartado Postal Casilla 160-C, Concepción. \\ *Autor Correspondiente, enrodriguez@udec.cl, Teléfono +56-041-2207324.
}

\begin{abstract}
RESUMEN
Los murciélagos representan el segundo orden de mamíferos más diverso. Sudamérica es rica en quirópteros. Sin embargo, Chile tiene una baja diversidad específica (12 especies) y ausencia de endemismos. Las publicaciones científicas que han abordado aspectos de la diversidad y taxonomía de estos mamíferos también son escasas. Esta revisión evaluó el estado actual del conocimiento de los quirópteros en Chile y sus principales resultados. Se distinguió de forma crítica los campos de investigación más y menos fortalecidos. Además se propone una explicación para la baja diversidad. Finalmente se confeccionó un mapa de distribución geográfica de las especies, relacionado con los biomas de Chile. Como resultado se recopilaron un total de 27 referencias en las últimas 2 décadas. El mayor número de publicaciones se alcanzó en la Infectologia y la Ecofisiología. El mapa de biomas mostró que la mayor diversidad se concentra en el Desierto de Atacama y presenta un patrón latitudinal decreciente. La taxonomía de los murciélagos chilenos ha variado fuertemente en el tiempo, aunque con periodos de estasis. Las principales limitantes a la diversidad de murciélagos en Chile poseen tanto un componente biogeográfico como un componente fisiológico de las mismas especies. Se necesita fortalecer los estudios macro y microevolutivos, de conservación y de bioacústica, para esclarecer importantes aspectos de la biología y diversidad de los murciélagos chilenos.
\end{abstract}

Palabras claves: Biomas, Chile, Conservación, Murciélagos, Patrones de Distribución.

\begin{abstract}
Bats are the second most diverse order of mammals. South America is rich in bats, however Chile has low species diversity (12 especies) and absence of endemism. Scientific publications that have addressed the biological diversity and taxonomy of these Chilean mammals are also scarce. This review assessed the state of knowledge of bats in Chile and its main results. We critically characterize those knowledge areas more developed and those that require further work. Additionally, we propose an explanation regarding this low diversity. Finally, we make a map that organizes bat diversity according to natural biomes of Chile. As results, we find 27 publications in the last two decades. These works are mainly focused on Infectology and Ecophysiology. The Chilean biome concentrating the highest bat diversity is the Atacama Desert, and the diversity is decreasing latitudinally. In spite of long periods of stasis, the taxonomy of Chilean bats abruptly changed in the last century. We propose a biogeographical and ecophysiological interaction for the observed patter of diversity. Finally, we stressed the importance of further micro and macroevolutionary research, as well as conservation and bioacustic studies, for better understanding of important issues still unsolved.
\end{abstract}

KeYwords: Bats, Biomes, Chile, Conservation, Distribution Patterns.

\section{INTRODUCCIÓN}

Los quirópteros representan aproximadamente el $20 \%$ de todas las especies de mamíferos en el mundo, siendo el segundo orden más numeroso de esta Clase, solo superado por Rodentia (Colin 2000). Se caracterizan por ser los únicos mamíferos realmente voladores (Findley 1993). Para este modo de desplazamiento presentan una serie de adaptaciones tanto en su anatomía interna como externa, pero manteniendo las características mamiferianas (e.g. glándulas mamarias, pelo, tres huesos en el oído medio, entre otros). 
Los quirópteros están ampliamente distribuidos en el mundo, pero ausentes en las regiones polares y porciones del territorio de Oceanía (Hill \& Smith 1984; Vaughan et al. 2000). La mayor cantidad de especies está concentrada en los trópicos (Findley 1993) y su diversidad disminuye a latitudes altas (Patterson et al. 2003). Sudamérica es rica en murciélagos. Países como Colombia poseen más del 50\% de las familias de murciélagos del Mundo, con 61 géneros y 198 especies siendo un grupo de inmensa importancia en la dinámica de los bosques tropicales (Solari et al. 2000). Perú es el tercer país del Nuevo Mundo con mayor diversidad de mamíferos (luego de Brasil y México, así como quinto en el mundo). La diversidad de quirópteros es igualmente alta, alcanzando las 8 familias, 63 géneros y 168 especies, aunque solo 7 son endémicas (Pacheco et al. 2009). Bolivia también posee alta diversidad de murciélagos con 132 especies (Aguirre et al. 2009, 2010), una parte importante amenazada por la destrucción de hábitat (Aguirre 1999). En Argentina la diversidad de especies decae a 63 especies incluidas en 4 familias (Barquez \& Díaz 2009). En Chile el número de especies desciende de forma drástica, se mantiene igual número de familias que en Argentina (4), pero reúnen a 12 especies (Díaz et al. 2011). Esta reducción en diversidad es consistente con el bajo número de especies de mamíferos en general (Palma 2007; Pacheco et al. 2009). También es importante destacar la ausencia de especies endémicas de quirópteros, siendo todas compartidas con países colindantes (Argentina, Bolivia y Perú).

En Chile, y en América en general, todos los murciélagos pertenecen al suborden Microchiroptera. Las 4 familias representantes en el país son: a) Phyllostomidae Gray 1825 con dos especies: Desmodus rotundus Geoffroy 1810, el único murciélago hematófago de Chile y Platalina genovensium Thomas 1928; b) Furipteridae Gray 1866 con un solo representante: Amorphichilus schnablii (Peters 1877); c) Vespertilionidae Gray, 1821 la familia más diversa en el país con 7 especies: Myotis chiloensis (Waterhouse 1838) y M. atacamensis Lataste 1821; Histiotus montanus (Philippi \& Landbeck 1861), H. macrotus (Poepigg 1835); e H. magellanicus (Philippi 1866); Lasiurus varius (Poeppig 1835) y L. cinereus (Palisot de Beauvois 1796); y d) Molossidae Gill 1872 con dos especies: Tadarida brasiliensis (Geoffroy 1824) y Mormopterus kalinowskii (Thomas 1893) (Modificado de Canals \& Cattan 2008; Iriarte 2008). Una parte importante de estas especies se restringe a la zona norte del país (e.g. P. genovensium), mientras que tan solo 4 especies alcanzan las zonas más australes de Chile (e.g. M. chiloensis).

La literatura que ha abordado la diversidad y taxonomía de los quirópteros chilenos es escasa y, en algunos casos, de larga data destacándose los aportes de Cabrera (1903; Catálogo descriptivo de los Quirópteros chilenos );
Osgood (1943; The mammals of Chile Zoological series); Mann (1978; Los pequeños mamíferos de Chile); Gantz y Martínez (2000) en el capítulo del libro de Muñoz y Yáñez (Mamíferos de Chile); Canals y Cattan (2008) en su libro "Radiografía a los murciélagos de Chile", brevemente en el libro "Los mamíferos de Chile" de Iriarte (2008) y en la Clave de identificación de los murciélagos del cono sur de Sudamérica de Díaz et al. (2009). Mientras que el número de investigaciones desarrolladas sobre quirópteros chilenos y publicados en revistas científicas, en los últimos años, no han sido especialmente numerosos, fenómeno tal vez propiciado por el bajo número de especies y ausencia de endemismos. Es muy posible que debido a estas condiciones naturales, el escaso conocimiento de este importante grupo de mamíferos haya permeado aún menos al quehacer económico y a las entidades gubernamentales que tratan con la biodiversidad. De esta manera, sólo en los últimos años se ha tratado a los murciélagos como parte, aunque no fundamental, de los estudios de línea de base de diversidad para los estudios de impacto ambiental de Chile (SAG 2012).

Esta revisión tiene como objetivo principal evaluar el avance del conocimiento de los quirópteros en Chile. Qué áreas de su biología son las más profundizadas y cuáles son sus principales resultados. Distinguiendo también, cuáles son los campos de investigación que deben ser ampliados y/o fortalecidos a futuro. Además, se recopila y condensa el conocimiento taxonómico de las especies en Chile, y se busca una explicación a la baja diversidad de especies en comparación con otros países de Sudamérica. Finalmente se entrega un mapa de distribución, como una herramienta para la correcta aplicación de metodologías de evaluación de impacto ambiental, en áreas ricas (proporcionalmente) de murciélagos en Chile.

\section{MATERIALES Y MÉTODOS}

Base de Datos sobre los Quirópteros Chilenos

Se generó una base de datos de publicaciones científicas sobre los quirópteros que habitan el territorio chileno realizadas recientemente (23 años), a través de búsquedas electrónicas mediante el buscador Google académico (http:// scholar.google.cl/) y a través de la plataforma de ISI Web of Science (http://thomsonreuters.com/web-of-science/). La búsqueda se restringió entre los años 1990 y 2013. Se utilizaron las palabras claves "chilean bats", "bats Chile", "Chiroptera Chile", "Murciélagos de Chile". La revisión bibliográfica también consideró monografías, capítulos de libro y libros recientes (e.g. Muñoz \& Yáñez 2000; Canals \& Cattan 2008; Iriarte 2008; Díaz et al. 2009), así como clásicos (e.g. Cabrera 1903; Osgood 1943; Mann 1978). 
Distribución de los Quirópteros de Chile

Para identificar la distribución de la diversidad de murciélagos en los diferentes biomas de Chile, se realizó un mapa de distribuciones que consideró el territorio político de Chile. Éste fue dividido a través del criterio de biomas (Olson et al. 2001) con el programa ArcMap 9.3 (ESRI 2009), la información de los rangos de distribución fue determinada según la base de datos de "La lista roja de la IUCN" (IUCN 2010), complementándose con la base bibliográfica del punto anterior. La complementación de datos individuales permitió desarrollar, por primera vez, un mapa que considera la totalidad de especies, su distribución y el tipo de bioma en el que éstas ocurren en Chile.

\section{RESULTADOS}

\section{A) TASA DE PUBLICACIÓN Y LÍNEAS DE INVESTIGACIÓN}

Se recopilaron un total de 27 referencias, en las últimas 2 décadas (1,2 publicaciones por año). Las líneas de investigación desarrolladas en estos trabajos han sido: la Bioacústica; la Ecofisiología; la Infectología; la Parasitología, Diversidad y Conservación. El mayor número de publicaciones se ha alcanzado en las áreas de investigación de la Infectología $(\mathrm{n}=11)$ y la Ecofisiología $(\mathrm{N}=9)$ (Fig. 1).
La publicación de trabajos en revistas científicas, cuyo foco sean los quirópteros chilenos, ha sido escasa (Fig. 1). En las últimas 2 décadas, 27 trabajos han sido publicados y de ellos 11 se han enfocado en la infectología del virus de la Rabia, 9 en ecofisiología, 3 en parasitología, 2 en bioacústica, 1 en diversidad y 1 en conservación. Esta información y sus detalles está disponible en el Anexo I.

\section{INFECTOLOGÍA}

La línea de investigación de infectología en murciélagos chilenos ha estado completamente ligada al virus de la Rabia. Esta disciplina se ha centrado en la epidemiologia del virus en Chile y en el efecto de la fauna de quirópteros en la transmisión de la enfermedad a animales domésticos y humanos (Favi \& Durán 1991; Favi \& Ramírez 1996). Estos estudios sugieren que a diferencia de lo acontecido en América Latina, en Chile el principal vector y reservorio de la Rabia no es la especie hematófaga $D$. rotundus, sino el insectívoro T. brasiliensis (Favi et al. 1999; Laval 2003: Laval \& Lepe 2008). Esta especie presenta la mayor prevalencia de rabia tanto en la Región Metropolitana (Santiago, Chile Mediterráneo; Favi et al. 2011) como en los centros urbanos y rurales del resto del país (Favi et al. 2008; Yung et al. 2012). Además, se han realizado tipificaciones genéticas y antigénicas de los virus de la rabia asociados a murciélagos en Chile (Yung et al. 2002, Yung et al. 2012), determinándose la presencia de múltiples variantes antigénicas mantenidas por diferentes especies de murciélagos.

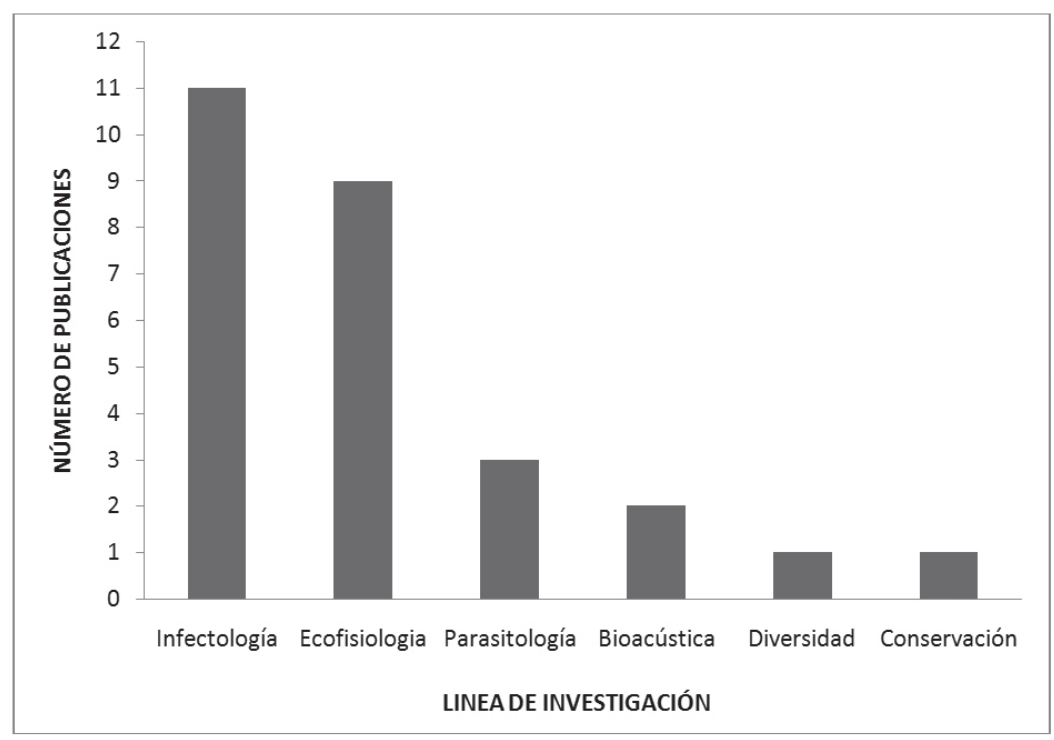

Figura 1. Número de publicaciones científicas según línea de investigación enfocadas en parte o totalmente en murciélagos chilenos. Rango temporal entre los años 1990 y 2013.

Figure 1. Number of scientific publications for research line focused in part or entirely in Chilean bats. Temporal window from 1990 to 2013. 
ECOFISIOLOGÍA

Las publicaciones de estudios ecofisiológicos de murciélagos chilenos, han seguido diversas líneas. Una parte está centrada en el estudio de la morfología alar, fuertemente ligada a la biomecánica y energética del vuelo y sus aspectos ecológicos y conductuales (Canals et al. 2001, Iriarte et al. 2002, Canals et al. 2005a). De este grupo, el trabajo con el mayor número de especies (8 especies) es el de Canals y colaboradores (2005a). Sus principales resultados indican que es posible encontrar cuatro grandes grupos de murciélagos chilenos: 1) los de alta carga alar y baja envergadura, cuyo vuelo es de alta agilidad, rápido, y de consumo moderado de energía (e.g. D. rotundus); 2) los de alta velocidad de vuelo y bajo consumo (e.g. Molossidae); 3) los de alta maniobrabilidad y baja velocidad que incluye a la mayoría de los vespertiliónidos; y 4) finalmente los de alta velocidad y agilidad como $L$. cinereus. Otra línea, estrechamente relacionada con la anterior, ha sido el estudio anatómico-comparativo del tamaño relativo del corazón y los pulmones entre pequeños mamíferos no voladores y murciélagos chilenos (Canals et al. 2005b). Los resultados indican un mayor tamaño relativo del corazón y los pulmones de los murciélagos como consecuencia directa del costo energético que significa el vuelo. También se han estudiado otros aspectos fisiológicos como la optimización funcional en las vías respiratorias del murciélago T. brasiliensis (Canals et al. 2005c; Canals \& Veloso 2008), así como los parámetros hematológicos, pulmonares, las demandas energéticas del vuelo y la correlación de ambos rasgos con la capacidad de difusión de oxígeno en los pulmones de las especies $M$. chiloensis y T. brasiliensis (Canals et al. 2007; Figueroa et al. 2007). Recientemente, se ha propuesto un modelo conjunto de las adaptaciones biomecánicas, respiratorias y cardiovasculares de murciélagos, usando como modelo principalmente especies chilenas (ver Canals et al. 2011).

\section{OTRAS LÍNEAS DE INVESTIGACIÓN}

Las publicaciones cuyos modelos de estudio son murciélagos chilenos y cuyas líneas son la Parasitología, Bioacústica, Diversidad, Conservación y Ecología han sido escasas ( $n=7)$. En Parasitología, se han estudiado las faunas parasitarias de T. brasiliensis (Muñoz et al. 2003, 2011) y de H. montanus (Muñoz et al. 2001). En Bioacústica, el trabajo de Ossa y colaboradores (2010) fue el primer estudio de ecolocalización realizado en murciélagos chilenos (M. chiloensis). A partir de los resultados obtenidos, los autores enfatizan la importancia de generar una sonoteca de las distintas especies de murciélagos presentes en Chile, debido a la importancia que tendría en la determinación más exacta de la distribución y la conservación de las mismas. Quizás siguiendo estas propuestas, en el reciente trabajo de Rodríguez-San Pedro \& Simonetti (2013), se identifican llamadas de ecolocalización de cuatro especies de murciélagos (T. brasiliensis, $M$. chiloensis, $H$. montanus y
L. varius), las que permitirán la identificación de especies en el campo sin necesidad de capturas y sus potenciales riesgos para los ejemplares. Finalmente, en estudios de biodiversidad y de conservación de vertebrados de Chile, se mencionan, de forma muy somera, a los murciélagos sin ahondar en detalles más allá del número de especies y sus potenciales amenazas (Mella et al. 2002; Jaksic \& Castro 2010).

\section{B) Biogeografía de los Quirópteros Chilenos}

Respecto a la distribución de las especies de quirópteros de Chile, el mapa de distribución y biomas (Fig. 2) es especialmente útil para visualizar como disminuye la diversidad a medida que aumenta la latitud y cuales biomas son los más diversos. La mayor parte de especies está presente en el Bioma del Desierto de Atacama (8 de las 12 especies). Este bioma alberga dos especies que se distribuyen exclusivamente en él ( $P$. genovensium y A. schnablii), y seis especies que habitan en el Desierto de Atacama como parte de su rango distribucional ( $D$. rotundus, $M$. atacamensis, $H$. montanus, $H$. macrotus, $T$. brasiliensis y $M$. kalinowskii). Mientras que 9 especies se encontraron en el Bioma del Matorral Chileno ( $D$. rotundus, M. chiloensis, M. atacamensis, H. montanus, H. macrotus, H. magellanicus, L. varius, L. cinereus y $T$. brasiliensis). Seguido del Bioma del Bosque Valdiviano con 7 especies (M. chiloensis, H. montanus, H. macrotus, H. magellanicus, L. varius, L. cinereus y T. brasiliensis). Con menor diversidad de especies se encuentran los Biomas de Los Andes centrales, con 5 especies (D. rotundus, $M$. atacamensis, $H$. macrotus, $T$. brasiliensis y M. kalinowskii). El Sur de la Estepa Andina, con 5 especies (D. rotundus, $H$. montanus, H. macrotus, H. magellanicus y T. brasiliensis) y finalmente el Bioma del Bosque Subpolar magallánico, el más austral, con 4 especies (M. chiloensis, $H$. montanus, $H$. magellanicus y L. varius).

\section{DISCUSIÓN}

A) BREVE Historia del CONOCIMIENTO TAXONÓMICO El número de especies de murciélagos en Chile no ha sido estable en el tiempo, registrándose variaciones importantes. Inicialmente, Cabrera (1903) reconoce la ocurrencia de 8 géneros en el país, que agrupan aproximadamente 17 especies. De ellas, 4 no han vuelto a ser documentadas en Chile: Lasiurus ega (Gervais, 1856), Lasiurus blossevillii frantzii (Peters, 1871), Glossophaga soricina (Pallas, 1766) y Sturnira lilium (Geoffroy, 1810). Otra parte importante corresponde a sinonimias. Según Osgood (1943) las siguientes especies mencionadas por Cabrera (1903) son sinónimas de otras especies documentadas para Chile: Vespertilio velatus Philippi 1861, es sinónimo de la especie Histiotus macrotus; Vespertilio montanus Philippi \& Landbeck 1861, Vespertilio magellanicus Philippi 1866 
y Vespertilio capucinos Philippi 1866, son sinónimos de la especie Histiotus montanus; Lasiurus grayi Tomes 1857 es sinónimo de Lasiurus cinereus; Myotis gayi (Lataste 1891) es sinónimo de Myotis chiloensis; y Nyctinomis brasiliensis Geoffroy 1824 es sinónimo de Tadarida brasiliensis. Del mismo modo, Mann (1978) indica que la especie Desmodus ruffus Wield 1824 (reconocida por Cabrera 1903), es un sinónimo de Desmodus rotundus.

Osgood (1943) reconoce 7 especies. Este autor considera a Myotis atacamensis como sinónimo de Myotis chiloensis, taxones que Cabrera (1903) considera especies distintas. Además no considera a Amorphochilus schnablii, Platalina genovensium y Mormopterus kalinowskii como presentes en el territorio chileno. Mientras que Mann (1978) y Gantz y Martínez (2000) coinciden en la ocurrencia de 9 especies, incorporando en sus trabajos a las especies $A$.schnablii y $M$. kalinowskii. Actualmente en Chile se consideran 12 especies, como consecuencia de una adecuación distribucional y otras dos modificaciones taxonómicas. La modificación distribucional guarda relación con la expansión del rango de distribución de la especie peruana $P$. genovensium hasta la Región de Arica y Parinacota (Galaz et al. 1999). Mientras que las modificaciones taxonómicas están relacionadas con: 1) el reconocimiento de los taxones $M$. chiloensis y $M$. atacamensis como especies distintas (Canals \& Cattan 2008; Iriarte 2008); 2) con la reciente elevación de la subespecie Lasiurus borealis varius presente en el país, a la categoría de especie (Pacheco et al. 2008); y 3) con el reconocimiento de la especie Histiotus magallenicus (Díaz et al. 2009), considerada un sinónimo de Histiotus montanus por Osgood (1943). Aunque en la mayoría de las publicaciones, incluso las más recientes, estas últimas modificaciones aún no han sido aplicadas (e.g. Favi et al. 2011; Canals et al. 2011).

\section{B) Diversidad de murciélagos en Chile}

La abundancia de quirópteros en Chile, al igual que en el resto de los mamíferos en general, es baja. Junto con Uruguay y Cuba, corresponden a los países latinoamericanos con menor número de especies de murciélagos (Gardner 2008). A pesar de ello, en Chile los quirópteros representan el cuarto orden con mayor número de especies, siendo superado por Carnivora (25 especies), Cetácea (42 especies) y Rodentia (ca. 68 especies aproximadamente). Esto implica que para la totalidad de los mamíferos chilenos, existe un porcentaje de endemismos bajo, reduciéndose a cero en quirópteros (Iriarte 2008; Diaz et al. 2009). Este patrón es totalmente contrastante con los altos niveles de diversidad observada en los países limítrofes. Diversas explicaciones se han propuesto para este patrón de disminución en la diversidad de mamíferos. Entre ellas la reducida superficie de área terrestre (comparado con el resto de los países vecinos), la heterogeneidad climática y la existencia de barreras naturales importantes, que pueden influir de forma negativa en la diversidad de especies, especialmente terrestres (Palma 2007).

Dentro de los límites de Chile, la mastozoofauna presenta un patrón de diversidad complejo. En general existe una mayor diversidad de mamíferos en el altiplano del extremo norte y la Patagonia del sur, mientras que la zona de menor diversidad es el Desierto de Atacama (Mella et al. 2005). Este patrón es totalmente contrastante con la diversidad de murciélagos encontrada, que decrece de Norte a Sur, siendo coincidente con lo documentado para el resto de los murciélagos del nuevo mundo (Patterson et al. 2003), pero con un máximo de diversidad en el bioma del Desierto de Atacama (Fig. 2).

Debido a esta particularidad del patrón de diversidad de los murciélagos chilenos, la explicación del mismo debe ser buscada en las características del paisaje natural de Chile. A pesar de su estrecha delgadez y su amplia extensión, Chile alberga 6 biomas distintos que son habitados por murciélagos. Esta heterogeneidad bioclimática puede representar una barrera importante para especies con características fisiológicas restrictivas y con bajas tasas metabólicas basales como los murciélagos insectívoros (Bozinovic \& Canals 2007), que corresponden a la mayoría de los murciélagos que habitan Chile y Sudamérica. Con respecto a la tasa metabólica basal de endotermos, se ha propuesto que una parte importante de su variación se explica por el tamaño corporal, mientras que el componente restante es histórico, lo que permite inferir la baja plasticidad del rasgo (Cruz-Neto \& Jones 2005). Una baja plasticidad, o lo que algunos autores denominan inercia filogenética (Blomberg \& Garland 2002; Frecklenton et al. 2002) implica una capacidad de adaptación reducida a cambios ambientales recientes (Harvey \& Pagel 1991).

Con estos antecedentes y considerando además el origen Neotropical de la mayoría de los linajes filogenéticos de murciélagos presentes en el sur de Sudamérica (e.g. Familias Furipteridae, Phyllostomidae; géneros Tadarida y Mormopterus; Lim 2009), se puede argumentar que parte importante de la geografía de Chile se presenta como un hábitat energéticamente marginal para Chiroptera. Esto se refleja en estrategias termorregulatorias como el sopor (Bozinovic \& Marquet 1991), el cual se observa en varias especies chilenas de rangos extremos (e.g. $L$. varius, $H$. montanus y $M$. chiloensis) como respuesta al excesivo gasto que implica habitar áreas donde las demandas termorregulatorias imponen déficits energéticos de largo plazo, o los recursos son altamente variables. En concordancia con lo anterior, las especies de quirópteros distribuidas exclusivamente en las áreas más diversas de Chile (biomas del Desierto de Atacama y de la Puna Seca de los Andes Centrales; Fig. 2), habitan áreas donde 
sus recursos y el hábitat térmico son similares a su rango de distribución principal. Por ejemplo, P. genovensium presenta una estrecha relación con un tipo de cactáceas presentes casi exclusivamente en Perú (Aragón \& Aguirre 2007) y zonas marginales de Chile, por tanto este recurso parece ser clave en su distribución geográfica y su ocurrencia. Finalmente, especies de distribución en el norte y centro de Chile, que potencialmente podrían habitar zonas australes, presentan hábitos y adaptaciones que son prohibitivas para tal efecto. En el caso de Desmodus rotundus su rango de distribución presenta como límite sur la zona del matorral central, específicamente las zonas costeras de Valparaíso (Canals \& Cattan 2008). Debido a su hábito hematófago es un murciélago que tiene poca capacidad de generar reservas lipídicas imposibilitando su hibernación cuando las condiciones ambientales se vuelven poco favorables, tal como pueden ser los inviernos fríos y de alta pluviosidad que son comunes al sur de su rango (Di Castri \& Hajek 1976; Gantz \& Martínez 2000). Por su parte la especie Tadarida brasiliensis, a pesar de tener la capacidad de caer en sopor en épocas más frías, es un murciélago habituado a climas secos, lo que se evidencia por su gran resistencia a la escasez de agua, facilitada por la facultad de concentrar la orina y evapotranspirar poco durante el vuelo (Iriarte 2008).

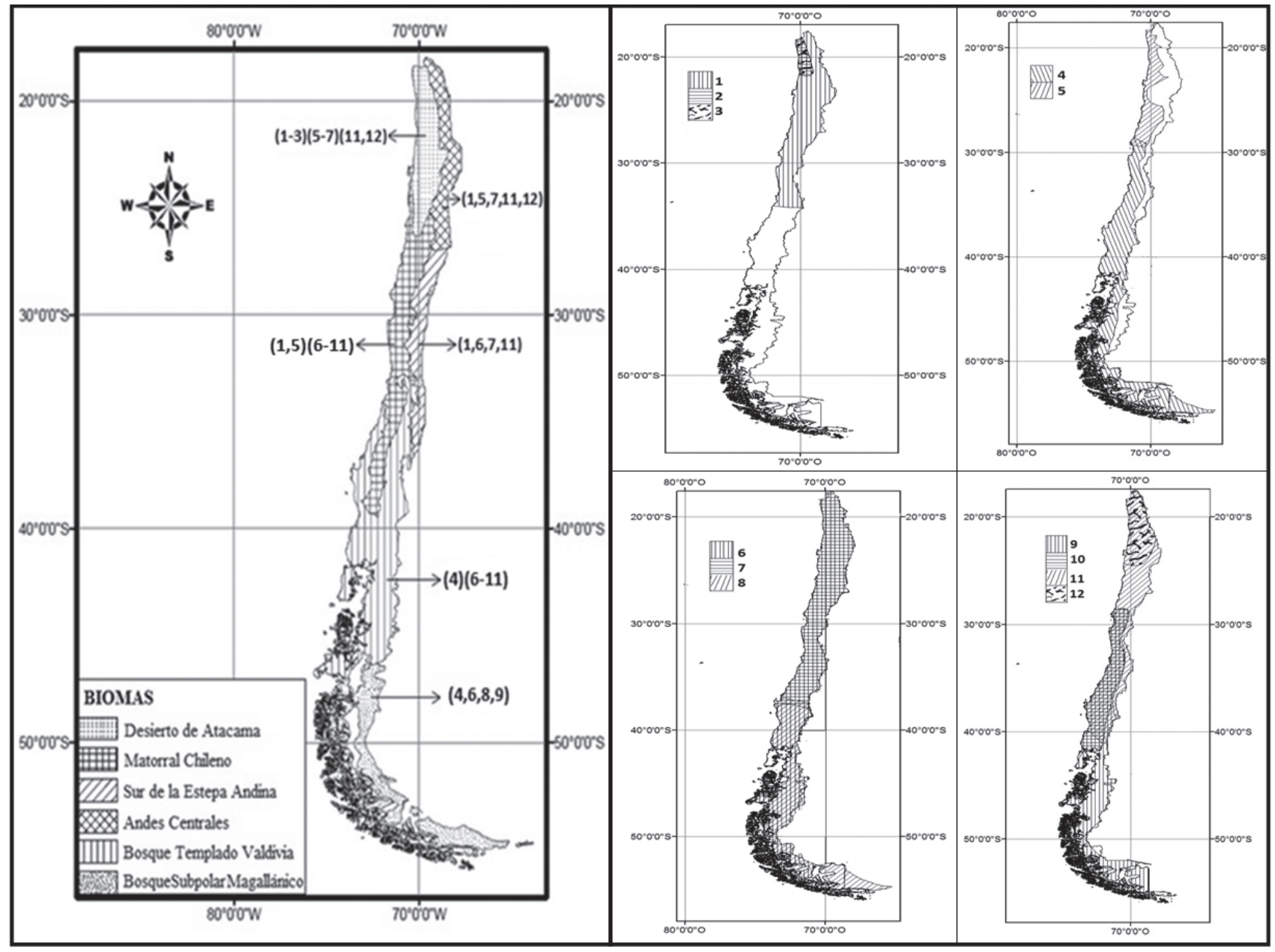

Figura 2. Territorio chileno dividido por biomas y mapa de Chile con la distribución de cada especie. Los números corresponden a: (1) D. rotundus, (2) P. genovensium, (3) A. schnablii, (4) M. chiloensis, (5) M. atacamensis, (6) H. montanus, (7) H. macrotus, (8) Histiotus magellanicus, (9) L. varius, (10) L. cinereus, (11) T. brasiliensis, (12) M. kalinowskii.

Figure 2. Map of Chile, divided by biomes, and map of Chile with the specific distribution. The numbers correspond to each of the species listed for Chile. (1) D. rotundus, (2) P. genovensium, (3) A. schnablii, (4) M. chiloensis, (5) M. atacamensis, (6) H. montanus, (7) H. macrotus, (8) Histiotus magellanicus, (9) L. varius, (10) L. cinereus, (11) T. brasiliensis, (12) M. kalinowskii. 
c) Futuras LíneAs de INVESTIGACión.

La revisión de las líneas de investigación actuales desarrolladas en los quirópteros de Chile demostró que, a pesar de todas las limitaciones, existe un conocimiento importante de algunas especies, ya sea por su rol en la transmisión de rabia (T. brasiliensis) o por su importancia como sujetos de estudio para comprender la naturaleza y complejidad del vuelo (e.g. Canals et al. 2005a). Aun así, existe un déficit sustancial en la investigación de varias especies de quirópteros.

$\mathrm{Si}$ bien las especies más septentrionales de Chile corresponden a formas que viven en zonas limítrofes $(P$. genovensium y M. kalinowskii), esta misma condición puede ser relevante para estudios de adaptación en ecofisiología, y coevolución en el caso de $P$. genovensium.

Con respecto a estudios que apunten a la sistemática y biodiversidad de los quirópteros chilenos es esencial realizar estudios filogenéticos y/o filogeográficos de $M$. chiloensis y $M$. atacamensis, cuyo estatus ha sido históricamente complejo, y actualmente intrigante. Al respecto, Cabrera (1903) identifica a estos taxones como dos especies distintas. Posteriormente ambas especies son sinonimizadas por Osgood (1943), permaneciendo en este status taxonómico por un tiempo considerable. Reciéntemente, se reconstruyó la filogenia del género Myotis utilizando datos moleculares (Larsen et al. 2012). Los resultados recapitulan a estas formas como componentes de clados filogenéticamente distantes donde $M$. atacamensis resulta parte de un clado que incluye especies caribeñas y centroamericanas, mientras que $M$. chiloensis se encuentra emparentado con especies exclusivamente sudamericanas (Larsen et al. 2012). Es interesante preguntarse, ¿Por qué dos especies, del mismo género, con rangos contiguos, pertenecen a clados tan distintos? Desde un punto de vista biogeográfico ¿sería más probable que estas especies fueran hermanas, o por lo menos pertenecieran al mismo clado? Si los resultados obtenidos por estos autores, son corroborados a escala geográfica más fina, ¿qué procesos biogeográficos habrían facilitado la dispersión de una especie cuyo ancestro es centroamericano o caribeño al norte de Chile y sur del Perú? Alternativamente, y apelando a la entidad taxonómica dudosa de $M$. atacamensis, es probable, que el ejemplar catalogado como tal en el trabajo de Larsen y colaboradores (2012) no sea realmente $M$. atacamensis. En este caso, lo ideal sería contar con un espécimen directamente colectado de la localidad típica (San Pedro de Atacama, Chile; Lataste 1892). El conflicto M. chiloensis/M. atacamensis, si bien es un ejemplo paradigmático, genera la expectativa de develar diversidad críptica dentro de los murciélagos de Chile.

Finalmente, es necesario realizar investigaciones con fines de conservación en especies que han sido declaradas amenazadas (e.g. A. schnablii IUCN: EN, Barquez \& Diaz 2008). Con mayor énfasis en aquellas que serán amenazadas con el desarrollo de la energía eólica en Chile. Existe evidencia importante de un impacto negativo, directa o indirectamente, sobre murciélagos por instalaciones de este tipo en EEUU (Kunz et al. 2007). Es importante analizar los efectos adversos de las plantas eólicas en la demografía de los murciélagos, y con ello generar mecanismos para minimizarlos (e.g. Escobar et al. 2015). Motivo más que suficiente para ingresar a estos mamíferos en los estudios de impacto ambiental de las instalaciones de esta naturaleza, considerando el enorme crecimiento de los parques eólicos $\mathrm{y}$ futuros proyectos para su proliferación en el centro-sur de Chile (MINENERGIA 2012).

\section{CONCLUSIONES}

Las principales conclusiones en este trabajo están enfocadas al estudio histórico, reciente y a la diversidad de murciélagos en Chile.

1. El grueso de la información de historia natural conocida respecto a los murciélagos en Chile se encuentra en textos clásicos (e.g. Osgood 1943; Mann 1978).

2. Las principales limitantes a la diversidad de murciélagos en Chile, poseen tanto un componente biogeográfico, como un componente fisiológico de las mismas especies.

3. El patrón de diversidad de murciélagos en Chile es latitudinal decreciente. Siendo el Bioma de mayor diversidad el del Desierto de Atacama.

4. Las áreas de investigación más desarrolladas guardan relación con la Infectología del virus de la Rabia y la Ecofisiología.

5. Existe un déficit importante del conocimiento en la conservación de mamíferos voladores en Chile, y en la Biología de especies de rangos distribucionales reducidos en el País (e.g. P. genovensium y A. schnablii).

6. Es necesario realizar estudios micro y macroevolutivos para lograr determinar si los taxones $M$. chiloensis y $M$. atacamensis en Chile, corresponden al mismo taxón, o representan especies distintas.

7. Se debe potenciar la realización de estudios bioacústicos. Los mismos deben incorporarse en la determinación de los rangos de distribución de las especies de quirópteros, para lograr catálogos más completos y precisos.

8. Es necesario evaluar los impactos de las plantas generadoras eólicas en la demografía de varias especies de murciélagos e incluirlos en los estudios de impacto ambiental.

\section{AGRADECIMIENTOS}

Los autores agradecen al proyecto de la Dirección de 
Investigación de la Universidad de Concepción DIUC 213.113.085-1AP y a la Beca de Magister de CONICYT.

\section{BIBLIOGRAFÍA}

Aguirre, L.F. 1999. Estado de conservación de los murciélagos de Bolivia. Chiroptera Neotropical 5:108-112.

Aguirre, L. F., Vargas A. \& Solari, S. 2009. Clave de campo para la identificación de los murciélagos de Bolivia. Centro de Estudios en Biología Teórica y Aplicada. Cochabamba, 38 pp.

Aguirre, L. F., Mamani, C.J., Barboza-Marque, C. \& MantillaMeluk H. 2010. Lista actualizada de los murciélagos de Bolivia. Revista Boliviana de Ecología y Conservación Ambiental 27:1-7.

Aragón, G. \& Aguirre, M. 2007. Conservación, distribución y densidad poblacional de Platalina genovensium (Thomas, 1928) en las Lomas del Morro Sama, distrito de Sama, Provincia de Tacna. Zonas Áridas 11:219-232.

Barquez, R. \& Diaz, M. 2008. Amorphochilus schnablii. The IUCN Red List of Threatened Species. Version 2014.1. $<$ www.iucnredlist.org>. Downloaded on 20 July 2014.

Barquez, R. M. \& DíAz, M.M. 2009. Clave de Identificación de los Murciélagos de Argentina. Publicación Especial $N^{\circ} 1$, PCMA (Programa de Conservación de los Murciélagos de Argentina), Editorial Magna, Tucumán, Argentina, 80 pp.

Blomberg, S.P. \& Garland, T. 2002. Tempo and mode in evolution: phylogenetic inertia, adaptation and comparative methods. Journal of Evolutionary Biology 15:899-909.

Bozinovic, F. \& Canals, M. 2007. Fisiología ecológica de mamíferos: compromisos y restricciones en el uso de la energía. En: Mamíferos de Chile Muñoz (Eds. Pedreros, A. \& J.L.Yañez), pp. 267-287. Ediciones CEA, Valdivia, Chile.

Cabrera, A. 1903. Catálogo descriptivo de los Quirópteros chilenos. Revista Chilena de Historia Natural 7:278-308.

Canals, M., Iriarte-Díaz, J., Olivares, R \& Novoa, F. 2001. Comparación de la morfología alar de Tadarida brasiliensis (Chiroptera: Molossidae) y Myotis chiloensis (Chiroptera: Vespertilionidae), representantes de dos diferentes tipos de vuelo. Revista Chilena de Historia Natural 74:699-704.

Canals, M., Grossi, B., Iriarte-Díaz, J. \& Veloso, C. 2005 a. Biomechanical and ecological relationships of wing morphology of eight Chilean bats. Revista Chilena de Historia Natural 78:215-227.

Canals, M., Atala, C. \& Grossi, B. 2005b. Relative size of hearts and lungs of several small bats. Acta Chiropterologica 7:65-72.

Canals, M., Atala, C., Olivares, R., Guajardo, F., Figueroa, D., Sabat, P. \& Rosenmann, M. 2005c. Functional and structural optimization of the respiratory system of the bat Tadarida brasiliensis (Chiroptera, Molossidae): Does the airway geometry matter? Journal of Experimental Biology 208:3987-3995.

Canals, M., Donoso, C., Figueroa, D \& Sabat, P. 2007. Pulmonary hematological parameters, energetic flight demands and their correlation with oxygen diffusion capacity in the lungs. Revista Chilena de Historia Natural 80:275-284.
Canals, M., Sabat, P. \& Veloso, C. 2008. The proximal airway of the bat Tadarida brasiliensis: a minimum entropy production design. Journal of Comparative Physiology 178:377-384.

Canals, M. \& Cattan, P. E. 2008. Radiografía a los murciélagos de Chile. Editorial Universitaria, Santiago, Chile. 107 pp.

Canals, M., Iriarte-Díaz, J. \& Grossi, B. 2011. Biomechanical, Respiratory and Cardiovascular Adaptations of Bats and the Case of the Small Community of Bats in Chile. En: Biomechanics in Applications (Ed. Vaclav Klika). ISBN: 978-953-307-969-1. URL: http://www.intechopen.com/ books/biomechanics-in-applications/biomechanicalrespiratory-and-cardiovascularadaptations-of-bats-andthe-case-of-the-small-community.

Colin, T. 2000. The Variety of Life. Oxford University Press, UK. $233 \mathrm{pp}$.

Díaz, M., Aguirre, L. \& Barquez, R. 2011. Clave de identificación de los murciélagos del cono Sur de Sudamérica. Centro de estudios en Biología Teórica y Aplicada. Cochabamba, Bolivia. 94 pp.

Di Castri, F. \& HajeK, E. 1976. Bioclimatología de Chile. Santiago: Universidad Católica de Chile, Chile. 160 pp.

Escobar, L. E., Juárez, C., Medina-Vogel, G. \& González, C. M. 2015. First Report on Bat Mortalities on Wind Farms in Chile. Gayana 79: 11-17.

ESRI (Environmental Systems Resource Institute). 2009. ArcMap9.2.ESRI, Redlands, California.

FAVI, M. \& DurÁn, J. 1991. Epidemiología de la rabia en Chile (1929-1988) y perspectivas en mamíferos silvestres. Avances en Ciencias Veterinarias 6:13-21.

FAVI, M. \& RAMírez, E.1996. Rabia humana en Chile. Laboratorio al día 12:7.

Favi, M., Yung, V., Pavletic, C., Ramírez, E., De Mattos, C.C. \& De Mattos, C.A. 1999. Rol de los murciélagos insectívoros en la transmisión de la rabia en Chile. Archivos de Medicina Veterinaria 31:157-65.

Favi, M., Rodríguez, L., Espinosa., C. \& Yung, V. 2008. Rabia en Chile.1989-2005. Revista Chilena de Infectología 25:8-13.

Favi, M., Bassaletti, A., López, J., Rodríguez, L. \& Yung, B. 2011. Descripción epidemiológica del reservorio de rabia en murciélagos de la Región Metropolitana. Chile. 20002009. Revista Chilena de Infectología 28:223-228.

Figueroa, D., Olivares, R., Salaberry, M., Sabat, P. \& Canals, M. 2007. Interplay between the morphometry of the lungs and the mode of locomotion in birds and mammals. Biological Research 40:193-201

FIndLeY, J.S. 1993. Bats, a community perspective. Cambridge University Press, Cambridge, UK. 167 pp.

Freckleton, R. P., Harvey, P.H. \& Pagel, M. 2002. Phylogenetic analysis and comparative data: a test and review of evidence. The American Naturalist 160:712-726.

Galaz, J. L., Torres-Mura, J. C., \& YÁñez, J. (1999). Platalina genovensium (Thomas, 1928), un quiróptero nuevo para la fauna de Chile (Phyllostomatidae: Glossophaginae). Noticiario Mensual del Museo Nacional de Historia Natural (Chile) 337:6-12.

Gantz, A. \& Martínez, D.R. 2000. Orden Chiroptera. En: Mamíferos de Chile (Eds. Muñoz-Pedreros, A \& J.L. Yañez), pp. 53-66. Ediciones CEA, Valdivia, Chile.

GARDNER, A.L. 2008. Mammals of South America, volume 
1: marsupials, xenarthrans, shrews, and bats (Vol. 1). University of Chicago Press. 669 pp.

Harvey, P.H. \& Pagel, M.D.1991 The Comparative Method in Evolutionary Biology. Oxford UniversityPress, Oxford. $248 \mathrm{pp}$.

Hill, J. \& Smith, J. 1984. Bats: A Natural History. Austin: University of Texas Press, Texas, USA. 877 pp.

Iriarte, A. 2008. Mamíferos de Chile. Lynx Ediciones, Barcelona, España. $221 \mathrm{pp}$.

Iriarte-Díaz, J., Novoa, F.F. \& Canals, M. 2002. Biomechanic consequences of differences in wing morphology between Tadarida brasiliensis and Myotis chiloensis. Acta Theriologica 47:193-200.

IUCN. 2010. The IUCN Red List of Threatened Species. Version 2010. URL: http://www.iucnredlist.org. Accesado: Abril, 2012.

JAKSIC, F. \& CASTRo, S. 2010. Ecología y biodiversidad de vertebrados de Chile: Análisis comentado de la Zoología de Claude Gay. Revista Chilena de Historia Natural 83:323-333.

Kunz, T.H., Arnett, E.B., Erickson, W.P., Hoar, A.R., Johnson, G.D., Larkin, R.P., Strickland, M.D., Thresher, R.W. \& TutTle, M.D. 2007. Ecological impacts of wind energy development on bats: questions, research needs, and hypotheses. Frontiers in Ecology and the Environment 5:315-324.

Larsen, R. J., Knapp, M. C., Genoways, H. H., Khan, F. A. A., Larsen, P. A., Wilson, D. E., \& Baker, R. J. 2012. Genetic diversity of neotropical Myotis (chiroptera: vespertilionidae) with an emphasis on South American species. PloS one 7(10): e46578.

Laval, E. 2003. La primera comunicación sobre rabia en Chile por el cirujano de la Armada don Pedro Videla Órdenes. Revista Chilena de Infectología 20:142-144.

Laval, E. \& LePe, P. 2008. Una visión histórica de la rabia en Chile. Revista Chilena de Infectología 25:2-7.

Lim, B. 2009. Review of the Origins and Biogeography of Bats in South America. Chiroptera Neotropical 15:391-410.

Mann, G. 1978. Los pequeños mamíferos de Chile. Gayana Zoología 40:1-342.

Mella, J., Simonetti., J., Spotorno, A. \& Contreras, L. 2002. Mamíferos de Chile. En: Diversidad y conservación de los mamíferos Neotropicales (Eds. Ceballos G. \& J. Simonetti ), pp. 151-183. CONABIO-UNAM, México.

MINENERGIA. 2012. Estrategia Nacional de Energía 2012-2030. Energía para el Futuro. Ministerio de Energía de Chile, Chile. 37 pp.

Muñoz, L., Aguilera, M. \& Casanueva, M. 2003. Prevalencia e intensidad de ectoparásitos asociados a Tadarida brasiliensis (Geoffroy Saint-Hilaire, 1824) (Chiroptera: Mollosidae) en Concepción. Gayana 67:1-8.

Muñoz, L., GonzÁlez, D. \& Fernández, I. 2001. Primer registro de Basilia silvae (Diptera: Nycteriibidae) sobre Histiotus montanus (Chiroptera: Vespertilionidae) en Chile. Gayana 65:221-222.
Muñoz, P., Fredes, F.,Raffo, E.,Gonzales-Acuña, D., Muñoz, L. \& CID, C. 2011. New report of parasite-fauna of the freetailed bat (Tadarida brasiliensis; Geoffroy, 1824) in Chile. Veterinary Research Communications 35:61-66.

Olson, D.M., Dinerstein, E., Wikramanayake E.D., Burgess, N.D., Powell, G.V.N., Underwood, E.C., D’Amico, J.A., Itoua, I., Strand, H.E., Morrison, J.C., Loucks, C.J., Allnutt, T.F., Ricketts, T.H., KuRA, Y., LamoreuX, J.F., Wettengel, W.W., Hedao, P. \& Kassem, K.R. 2001. Terrestrial Ecoregions of the World: A New Map of Life on Earth. BioScience 51:933-938.

OsGood, W.H. 1943. The mammals of Chile. Field Museum of Natural History, Zoological Series 30:1-268.

Ossa, G., Ibarra, J.T., Barboza, K., Hernández, F., Gálvez, N., LAKER, J. \& BONACIC, C. 2010. Analysis of the echolocation calis and morphometry of a population of Myotis chiloensis (Waterhouse, 1838) from the southern Chilean temperate forest. Ciencia e Investigación Agraria 37:131-139.

Pacheco, V., Aguirre, L. \& Mantilla, H. 2008. Lasiurus varius. En: IUCN 2013. IUCN Red List of Threatened Species. Version 2013.1. URL: www.iucnredlist.org. Accesado: Agosto 27, 2013.

Pacheco, V., Cardenilla, R., Salas, E., Tello, C. \& Zeballos, H. 2009. Diversidad y endemismo de los mamíferos del Perú. Revista Peruana de Biología 16:5-32.

Palma, R.E. 2007. Estado actual de la mastozoología en Chile. Mastozoología neotropical 14:5-9.

Patterson, B.D., Willig, M.R. \& Stevens, R.D. 2003. Trophic strategies, niche partitioning and patterns of ecological organization. En: Bat Ecology (Eds. Kunz, T.H. \& M.B. Fenton), pp. 536-579. The University of Chicago Press, Chicago.

Rodríguez-San Pedro, A. \& Simonetti, J. 2013. Acoustic identification of four species of bats (Order Chiroptera) in central Chile. Bioacoustics: The International Journal of Animal Sound and its Recording 22:165-172.

SAG. 2012. Pauta de Evaluación Ambiental Proyectos Lineales D-PR-GA-008. Ministerio de Agricultura. Chile. 12 pp.

Solari, S., Muñoz-Saba, Y., Rodríguez-Mahecha, J. V., Defler, T. R., Ramírez-Chaves, H. E., \& Trujillo, F. 2013. Riqueza, endemismo y conservación de los mamíferos de Colombia. Mastozoologia Neotropical 20:301-365.

Teeling, E.C., Springer, M.S., Madsen, O., Bates, P., O’Brien, S.J. \& Murphy, W.J. 2005. A molecular phylogeny for bats illuminates biogeography and the fossil record. Science 307:580-584.

Vaughan, T., Ryan, J. \& Czaplewski, N. 2000. Mammalogy, 4th Edition. Toronto: Brooks Cole. 565 pp.

Yung, V., Fernández, J. \& FAVI, M. 2002. Genetic and antigenic typing of rabies virus in Chile. Archives of Virology 147:197-205.

Yung, V., Favi, M. \& Fernandez, J. 2012. Typing of the rabies virus in Chile, 2002-2008. Epidemiology and Infection 140:2157-2162. 
Gayana 79(1), 2015

ANEXO I: Tabla de las referencias bibliográficas utilizadas para realizar la figura 1.

ANNEX I: Table showing the references used for Figure 1.

\begin{tabular}{|c|c|}
\hline Disciplinas & Referencia Bibliográficas \\
\hline Infectologia & $\begin{array}{l}\text { FAVI, M. \& DURÁN, J. 1991. Epidemiología de la rabia en Chile (1929-1988) y perspectivas en mamíferos } \\
\text { silvestres. Avances en Ciencias Veterinarias 6:13-21. } \\
\text { FAVI, M. \& RAMÍREZ, E.1996. Rabia humana en Chile. Laboratorio al día 12:7. } \\
\text { FAVI, M., YUNG, V., PAVLETIC, C., RAMÍREZ, E., DE MATTOS, C.C. \& DE MATTOS, C.A. 1999. Rol de los } \\
\text { murciélagos insectívoros en la transmisión de la rabia en Chile. Archivos de Medicina Veterinaria 31:157-65. } \\
\text { LOZA-RUBIO, E., DE MATTOS, C. C., AGUILAR-SETIÉN, A., \&DE MATTOS, C. A. 2000. Aislamiento y } \\
\text { caracterización molecular de un virus rábico, obtenido de un murciélago no hematófago en la ciudad de México. } \\
\text { Vet Mex, 31, 147-51. } \\
\text { YUNG, V., FERNÁNDEZ, J. \& FAVI, M. 2002. Genetic and antigenic typing of rabies virus in Chile. Archives of } \\
\text { Virology 147:197-205. } \\
\text { YUNG, V., FAVI, M. \& FERNANDEZ, J. 2012. Typing of the rabies virus in Chile, 2002-2008. Epidemiology and } \\
\text { Infection 140:2157-2162. } \\
\text { Laval, E. 2003. La primera comunicación sobre rabia en Chile por el cirujano de la Armada don Pedro Videla Órde- } \\
\text { nes. Revista Chilena de Infectología 20:142-144. } \\
\text { FAVI, M., RODRÍGUEZ, L., ESPINOSA., C. \& YUNG, V. 2008. Rabia en Chile.1989-2005. Revista Chilena de } \\
\text { Infectología 25:8-13 } \\
\text { LAVAL, E. \& LEPE, P. 2008. Una visión histórica de la rabia en Chile. Revista Chilena de Infectología 25:2-7. } \\
\text { FAVI, M., BASSALETTI, A., LÓPEZ, J., RODRÍGUEZ, L. \& YUNG, B. 2011. Descripción epidemiológica del } \\
\text { reservorio de rabia en murciélagos de la Región Metropolitana. Chile. 2000-2009. Revista Chilena de Infectología } \\
\text { 28:223-228. } \\
\text { YUNG, V., FAVI, M. \& FERNANDEZ, J. 2012. Typing of the rabies virus in Chile, 2002-2008. Epidemiology and } \\
\text { Infection 140:2157-2162 }\end{array}$ \\
\hline Ecofisiologia & $\begin{array}{l}\text { CANALS, M., IRIARTE-DÍAZ, J., OLIVARES, R \& NOVOA, F. 2001. Comparación de la morfología alar de } \\
\text { Tadarida brasiliensis (Chiroptera: Molossidae) y Myotis chiloensis (Chiroptera: Vespertilionidae), representantes de } \\
\text { dos diferentes tipos de vuelo. Revista Chilena de Historia Natural 74:699-704. } \\
\text { IRIARTE-DÍAZ, J., NOVOA, F.F. \& CANALS, M. 2002. Biomechanic consequences of differences in wing } \\
\text { morphology between Tadarida brasiliensis and Myotis chiloensis. Acta Theriologica 47:193-200. } \\
\text { CANALS, M., GROSSI, B., IRIARTE-DÍAZ, J. \& VELOSO, C. 2005. Biomechanical and ecological relationships } \\
\text { of wing morphology of eight Chilean bats. Revista Chilena de Historia Natural 78:215-227. } \\
\text { CANALS, M., ATALA, C. \& GROSSI, B. 2005. Relative size of hearts and lungs of several small bats. Acta } \\
\text { Chiropterologica 7:65-72. } \\
\text { CANALS, M., ATALA, C., OLIVARES, R., GUAJARDO, F., FIGUEROA, D., SABAT, P. \& ROSENMANN, M. } \\
\text { 2005. Functional and structural optimization of the respiratory system of the bat Tadarida brasiliensis (Chiroptera, } \\
\text { Molossidae): Does the airway geometry matter? Journal of Experimental Biology 208:3987-3995. } \\
\text { CANALS, M., DONOSO, C., FIGUEROA, D \& SABAT, P. 2007. Pulmonary hematological parameters, energetic } \\
\text { flight demands and their correlation with oxygen diffusion capacity in the lungs. Revista Chilena de Historia } \\
\text { Natural 80:275-284. } \\
\text { FIGUEROA, D., OLIVARES, R., SALABERRY, M., SABAT, P. \& CANALS, M. 2007. Interplay between the } \\
\text { morphometry of the lungs and the mode of locomotion in birds and mammals. Biological Research 40:193-201 } \\
\text { CANALS, M., SABAT, P. \& VELOSO, C. 2008. The proximal airway of the bat Tadarida brasiliensis: a minimum } \\
\text { entropy production design. Journal of Comparative Physiology 178:377-384. }\end{array}$ \\
\hline
\end{tabular}




\begin{tabular}{|c|c|}
\hline & $\begin{array}{l}\text { CANALS, M., IRIARTE-DÍAZ, J. \& GROSSI, B. 2011. Biomechanical, Respiratory and Cardiovascular } \\
\text { Adaptations of Bats and the Case of the Small Community of Bats in Chile. En: Biomechanics in Applications (Ed. } \\
\text { Vaclav Klika). ISBN: 978-953-307-969-1. URL: http://www.intechopen.com/books/biomechanics-in-applications/ } \\
\text { biomechanical-respiratory-and-cardiovascularadaptations-of-bats-and-the-case-of-the-small-community }\end{array}$ \\
\hline Parasitologia & $\begin{array}{l}\text { MUÑOZ, L., GONZÁLEZ, D. \& FERNÁNDEZ, I. 2001. Primer registro de Basilia silvae (Diptera: Nycteriibidae) } \\
\text { sobre Histiotus montanus (Chiroptera: Vespertilionidae) en Chile. Gayana 65:221-222. } \\
\text { MUÑOZ, L., AGUILERA, M. \& CASANUEVA, M. 2003. Prevalencia e intensidad de ectoparásitos asociados a } \\
\text { Tadarida brasiliensis (Geoffroy Saint-Hilaire, 1824) (Chiroptera: Mollosidae) en Concepción. Gayana 67:1-8. } \\
\text { MUÑOZ, P., FREDES, F.,RAFFO, E.,GONZALES-ACUÑA, D., MUÑOZ, L. \& CID, C. 2011. New report } \\
\text { of parasite-fauna of the free-tailed bat (Tadarida brasiliensis; Geoffroy, 1824) in Chile. Veterinary Research } \\
\text { Communications 35:61-66. }\end{array}$ \\
\hline Bioacustica & $\begin{array}{l}\text { OSSA, G., IBARRA, J.T., BARBOZA, K., HERNÁNDEZ, F., GÁLVEZ, N., LAKER, J. \& BONACIC, C. } 2010 . \\
\text { Analysis of the echolocation calis and morphometry of a population of Myotis chiloensis (Waterhouse, 1838) from } \\
\text { the southern Chilean temperate forest. Ciencia e Investigación Agraria 37:131-139. } \\
\text { RODRÍGUEZ-SAN PEDRO, A. \& SIMONETTI, J. 2013. Acoustic identification of four species of bats (Order } \\
\text { Chiroptera) in central Chile. Bioacoustics: The International Journal of Animal Sound and its Recording 22:165- } \\
\text { 172. }\end{array}$ \\
\hline Biodiversidad & $\begin{array}{l}\text { JAKSIC, F. \& CASTRO, S. 2010. Ecología y biodiversidad de vertebrados de Chile: Análisis comentado de la } \\
\text { Zoología de Claude Gay. Revista Chilena de Historia Natural 83:323-333. }\end{array}$ \\
\hline Conservación & $\begin{array}{l}\text { MELLA, J., SIMONETTI., J., SPOTORNO, A. \& CONTRERAS, L. 2002. Mamíferos de Chile. En: Diversidad } \\
\text { y conservación de los mamíferos Neotropicales (Eds. Ceballos G. \& J. Simonetti), 151-183. CONABIO-UNAM, } \\
\text { México }\end{array}$ \\
\hline
\end{tabular}

Recibido: 03.04.14

Aceptado: 15.04.15 